\title{
Nelder-Mead Optimized MADM Decision Support for Vertical Handover
}

\author{
Malathy E M, Vijayalakshmi Muthuswamy
}

\begin{abstract}
The vertical handover decision strategy is a challenging problem to support seamless mobility in different wireless technology. The handover decision process is reported to facilitate the required Quality of Service, but bad network selection and overload condition of the chosen network fallout in handover failures. Traditional handoff techniques typically are based on single parameter and are inefficient due to superfluous handovers. In addition, existing fuzzy based handoff method reduces the handover failures but increase the computational complexity. Furthermore, the existing mobile controlled handover techniques are not suitable for heterogeneous environment. Therefore this paper focus the need of efficient, robust and flexible vertical handover technique to reduce the handover failures and design an MADM based network controlled handover that maximize QoS in heterogeneous environment.
\end{abstract}

Index Terms - Vertical handover; decision support; Nelder Mead; MADM technique.

\section{INTRODUCTION}

The evolution of the next generation wireless network has led to the increasing demand for handheld devices to enjoy mobility through "Always Best Connected" services. In the next generation wireless network, seamless best-possible access to a network, which has a widely varying set of network characteristics, requires rigorous mobility management. The vertical handover facilitates users to roam across different networks with seamless network connection. The vertical handover process that supports mobility can be initiated as mobile controlled handoff or as a network controlled handoff process. Associating the user with a suitable wireless network using a vertical handover process through wide network integration is a challenging and difficult problem that has drawn the attention of many researchers.

Traditional mobile controlled handoff techniques [1] [2]typically are based on single parameter and are inefficient due to superfluous handovers. Multi Attribute Decision Making (MADM) technique is employed in the vertical handover process to select the most appropriate alternative network, but yields merely feasible results in a heterogeneous network. Fuzzy-based optimal method [3] discussed in the literature is computationally intensive due to the conflicting rules generated by the inconsistent information drawn from different sources leading to complex decision management.

In addition, various mobility management strategies [4] combined with MADM for QoS support discussed in the

Malathy E M, SSN College of Engineering, Kalavakkam, Chennai, T.N, India.

Vijayalakshmi Muthuswamy, College of Engineering, Anna University, Chennai, T.N, India.
Revised Manuscript Received on July 10, 2019.

literature are based on the mobilecontrolled handoff[ 5]. Such a design fails to select the best network since the device has limited power and information about the networks. Subsequently, the ping-pong effect also gradually increases as the mobile terminal chooses the network based on the previous selections. High complexity in the decision making process, the delay incurred, inappropriate routing metrics, lack of proactive congestion check mechanism in the candidate network that occur during the entire mobilecontrolled handoff process deteriorates the system efficient performance and hence needs critical attention. On the other hand, network controlled handover process is found to address merely mobile IP handover functionality. This brings significant performance improvement in admission policy and signaling problems that are present in homogeneous network[]. However, it fails to perform in the unreliable dynamic heterogeneous environment.

This research work aims to reduce the vertical handover failures and maximize handover service by developing an VIKOR- NMO ( Nelder Mead Optimization) technique. The proposed network controlled policy on optimal network selection caters to different traffic classes considering multiple QoS parameters. The handover is performed by the zone gateway of the network on behalf of the mobile device and hence removes the possible limitation of mobilecontrolled handoff process. The proposed vertical handover approach adopts three stage network-controlled handover process using MADM based network estimation and an enhanced optimization policy along with cooperative cache support. The basic first stage of the proposed work utilizes the parameter weight computation with the Analytic Hierarchy Process (AHP) to make an appropriate estimation for handover decision. The work then examines MADM method such VIsekriterijumsko KOmpromisno Rangiranje (VIKOR) to ensure selection of best candidate network based on traffic classes provided. In the second stage of the work, the estimated decision taken from the previous stage is utilized Nelder- Mead direct search method. Nelder-Mead method proposed to prevent unnecessary handovers that are caused due to uncertain conditions prevailing with mobile locations. Thus, this stage fine-tunes the decision policy to achieve an optimal number of handovers with optimal computation of QoS parameters.

\section{PROPOSED NELDER MEAD METHOD}

This section presents the discovery phase during handover and the collection of handover information. This process is 


\section{NELDER-MEAD OPTIMIZED MADM DECISION SUPPORT FOR VERTICAL HANDOVER}

effective in giving all information about different access point available, mobile terminals and other related parameters that are needed to proceed with handover. The required parameters are gathered to compute the handover decision. The proposed scheme measures the signal strength from the current PoA and the available candidate networks that efficiently avoids all the call drops due to fading condition that occurs in next generation network. The polynomial regression gives the accurate signal strength of data sequences with accumulator of previous data. Analytic Hierarchy Process (AHP) decomposes all major decision problems into a hierarchy of alternatives as stated by Saaty (1988). The evaluation parameters are computed from the scale value of weights and they play an important role in determining the network selection process. The aim of the method finds the value to be assigned for different traffic classes. The process brings clear picture of all the essential alternatives that influence the outcome of a decision. The QoS traffic classes include streaming, one-way transport classes such as watching video via YouTube where the delay is less important than the throughput. The background traffic is also a one-way traffic that includes sending or receiving SMS or emails. This class is of less importance to delay and throughput. Conversational traffic class on the other hand is a two way traffic for which online video conferencing is an ideal example, with delay as a very sensitive parameter, and throughput less important. Iterative traffic is also a two-way traffic class that includes chatting and truncations on web services. Thus, AHP fixes priority to the parameters to generate an accurate network between WiMAX and WiFi.

\section{MADM- VIKOR COMPUTATION MODEL}

VIKOR (VIsekriterijumsko KOmpromisno Rangiranje) is a MADM method that gives a compromise score of the network with the list of measurable alternatives that are present along with conflicting criteria (Parameshwaran et al. 2015). This is an aggregating deciding function, which produce results closest to the desirable ideal solution. VIKOR method in this research work is adopted as it gives the best result for voice data (Peng 2012). The method determines the best value $\mathrm{Fi}+$ and worst value $\mathrm{Fi}$ - for the evaluation criterion function. The objective function determination is the first step with evaluation attribute identification. The method determines the best value Fi+ and worst value Fi- for the evaluation criterion function.

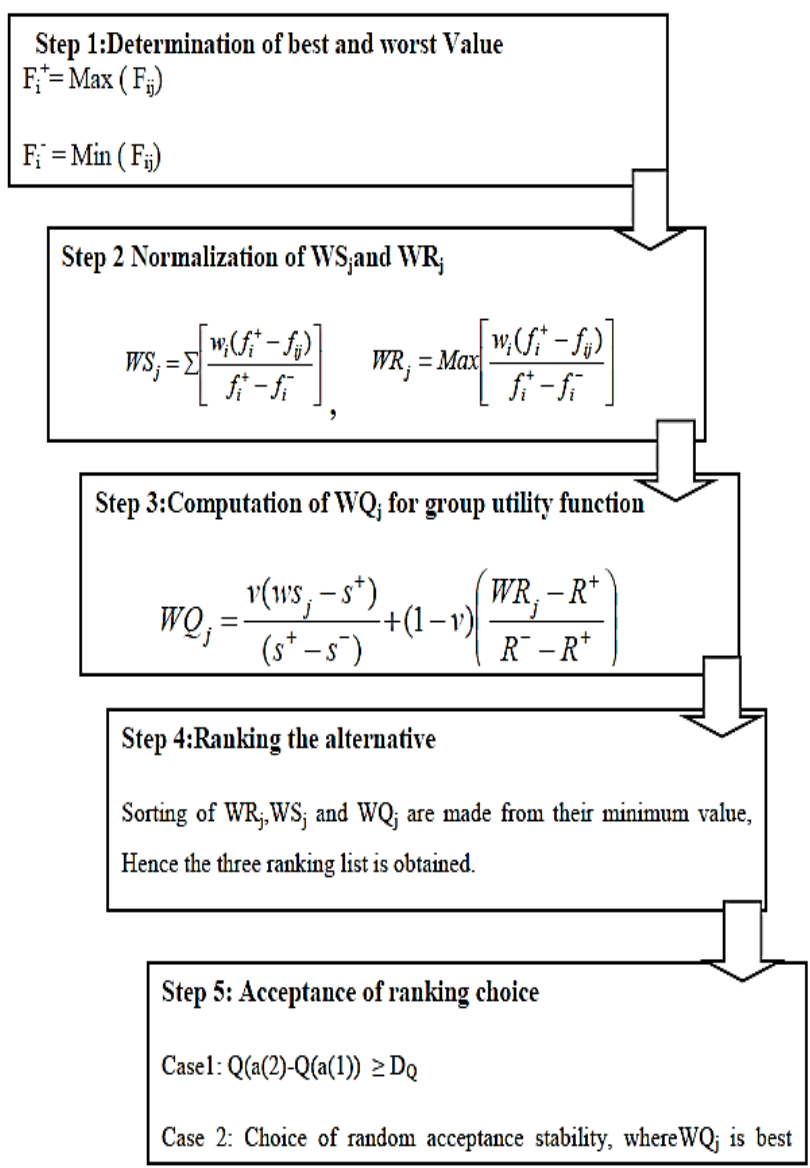

The method determines the best value $\mathrm{Fi}+$ and worst value Fi- for the evaluation criterion function. The objective function determination is the first step with evaluation attribute identification. The network ranking is obtained by sorting the score values of WSj, WRj, WQj by descending order. The VIKOR compromise network score is obtained by WQj measured value. The minimum WQj value brings the best alternative solution. The two solutions arise with case 1 , where $\mathrm{a}(1)$ and $\mathrm{a}(2)$ are of acceptable stage when complementary conditions are satisfied. With VIKOR, the computation of the vertical handover performed is measured as the occurrence of handover event. The collective ranking performed provides the best network selection to mobile nodes and QoS selection enable the moble user to effectivly use the services of network.

\section{A. Nelder Mead Optimization}

The Nelder-Mead method defined as a direct search optimization technique uses the concept of simplex $\mathrm{N}+1$ vertices in $\mathrm{N}$ dimension. The technique yields an optimal solution with $\mathrm{N}$ variable parameters for well-defined objective functions. The algorithm is designed to minimize the Function $f(x)$ value that creates the estimation value to trigger vertical handover. The energy consumption of a route due to handover of mobile nodes across the links present in a heterogeneous environment is defined as handover route energy. To effectively evaluate the handover energy consumption, handover transition energy alone is

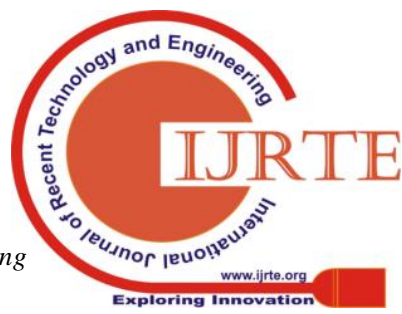


considered. Let there be $\Gamma$ links present in a route $R$ for the mobile node to switch over to candidate network. The $\Gamma$ links connect $\Gamma+1$ number of nodes including the current point of attachment to candidate network. Let BSi be the current point of attachment and BSc be the andidate network point of attachment for handover. Thus the handover route can be written as a finite sequence of links.

The objective is to improve the performance of the vertical handover decision in order to minimize the number of handovers and avoid unnecessary handovers. Hence, objective function starts with the worst point $\mathrm{N}(3)$ and moved towards $\mathrm{N}(1)$ and $\mathrm{N}(2)$. The points where the objective function values decrease at vertices are observed. At each step, the transformation is determined by computing one or more test points. The optimal solution that minimizes the function $\mathrm{F}(\mathrm{x})$ value is the estimation value that triggers vertical handover. The vertices that represent the mobile node position with respect to zone nodes are taken as $\mathrm{N}(1)$, $\mathrm{N}(2), \mathrm{N}(3)$. The iteration generates the vertex of mobile order with four possible operations reflection, expansion, contraction, and shrink. Reflection is computed the reflection point $\mathrm{Nr}$ by $\mathrm{N}_{\mathrm{i}}=\mathrm{V}+\rho\left(\mathrm{V}-\mathrm{N}_{\mathrm{n}+1}\right)$ if $\mathrm{f}(\mathrm{N} 1) \leq$ $\mathrm{f}(\mathrm{Nr})$ $\mathrm{f}(\mathrm{Nn})$.

If $\mathrm{f}(\mathrm{Nr})<\mathrm{f}(\mathrm{N} 1)$ then expansion is computed. If $\mathrm{f}(\mathrm{Nr}) \geq \mathrm{f}(\mathrm{xn})$ then contract operation is carried. These parameters and variable (represented in Table 6.1) are selected in such a way that fulfill $\rho>0, \chi>1,0<\gamma<1$, and $0<\sigma<1$.

\section{PERFORMANCE ANALYSIS OF NMO- VIKOR \& RESULTS}

To analyze the performance of the proposed Nelder-Mead optimization approach NS2 simulation is used. The method investigates the convergence rate of the objective function in order to study whether the proposed vertical handover decision optimize the energy consumption of the VHD. Variations in the speed of mobile users are performed to manipulate the handover occurrence rate. The mobility of the mobile nodes is varied with random distance with velocity varying from $10 \mathrm{~km} / \mathrm{h}$ to $50 \mathrm{~km} / \mathrm{h}$. Mobile node sample considered vary from 10 to 250 . The proposed work is network controlled and the optimal solution on vertical handover has to be initiated on the mobile nodes. The AODV protocol (Bikramjeet \& Brar 2013) makes limited periodic updates of mobile nodes to mobility management. In addition, this routing protocol affords a method that reduces overhead and guarantees loop freedom in the paths. Hence, AODV routing protocol is adopted for the simulation. The mobile nodes are deployed in 4 Point of attachment with two base stations in WiMAX network and two access points in WiFi network represented as PoA1, $\mathrm{PoA} 2, \mathrm{PoA} 3$ and PoA4 respectively. The single cell radius is $100 \mathrm{~m}$ in $\mathrm{WiFi}$ and $1500 \mathrm{~m}$ in WiMAX. The simulation parameters used are reported.

The interaction between WiFi and WiMAX is observed through the simulation. These networks are designed to promote interoperability localized in the network. All mobile nodes are heterogeneous and energy-constrained. The size of the data packet is fixed according to four different traffic classes. The service selected is one among the four type of classes, namely streaming, conversational,

background, and interactive traffic classes. Figure 1 represents the handover computation with an occurrence of vertical handover between the WiMAX and WiFi networks. The result is compared with the multi attribute TOPSIS method, where an increase in handover occurs because the optimization policy is not employed. The speed of the mobile devices is varied from $10 \mathrm{~km} / \mathrm{h}$ to $50 \mathrm{~km} / \mathrm{h}$ and handover occurrence is observed. The result is presented with average values. The result shows the number of handover occurrence, VIKOR technique and VIKOR Nelder-Mead Optimization (VIKOR-NMO). The average value is presented once all simulation is carried. The maximum handover occurrence observed with VIKOR method is $68 \%$ with WiMAX network and $47 \%$ in WiFi network with background traffic classes at speed of 10 $\mathrm{km} / \mathrm{h}$. Figure 1 shows the method having $47 \%$ VHD count in WiMAX network and $42 \%$ in with background traffic classes. Once the speed is varied executes heavy vertical handover decision with maximum of $56 \%$ occurrence for in WiMAX and $42 \%$ in WiFi network for background traffic classes.

In WiMAX network, 20\% improvement is noted with line of sight transmission of data with initial speed of about 10 $\mathrm{km} / \mathrm{h}$. It is also observed that $23 \%$ improvement is achieved in avoiding unnecessary handover at the speed of about 50 $\mathrm{km} / \mathrm{h}$ with WiFi network, it is $12 \%$ improvement in initial at speed and $16 \%$ at $50 \mathrm{~km} / \mathrm{h}$.

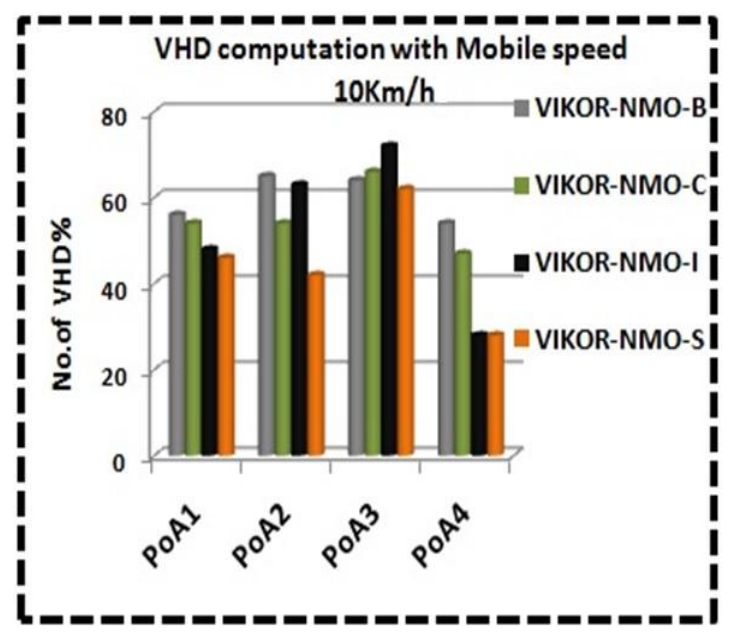

Figure 1 Handover computation

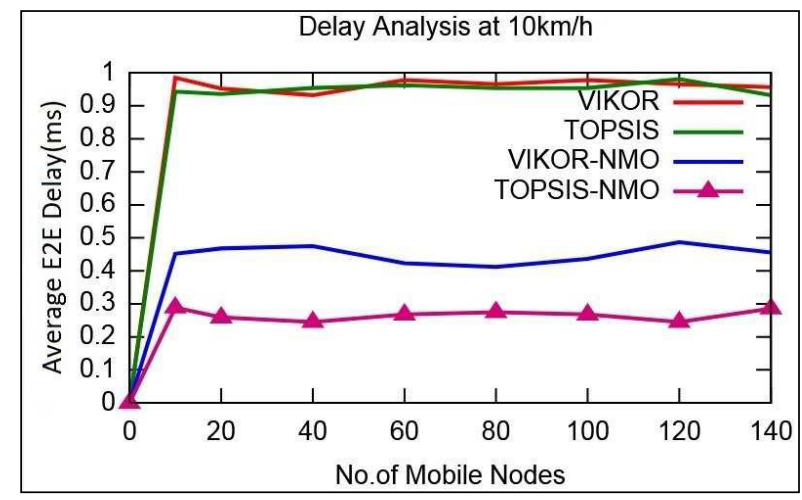

Figure 2 : Delay measure.

V. CONCLUSION 
VIKOR Nelder-Mead Optimization (VIKOR-NMO) for vertical handover in the next generation wireless network is proposed. The main objective of this proposed work is to design a simple decision scheme to minimize unnecessary handover and improve seamless handover with much reduced delay performance. In the proposed technique, multiple parameters are evaluated with an AHP method to identify the correct weight-age for VHD. VIKOR method is compared to bring the benefits of the ranking scheme along the optimization technique. The method determines energy consumption of different nodes and paths to implement optimization policy. For this, multiple vertices of a mobile node are selected and optimal location is computed by Nelder-Mead direct search method at different convergent timings. The output effectiveness and benefits of the proposed technique are studied based on the metrics considered. Simulation evaluation, the handover result is found to be effective when compared to MADM vertical handover scheme. VIKOR-NMO. Also, the statistical analysis demonstrates that delay measure outperforms with multiple load in the network in VIKOR NMO by 0.3 second. Therefore, this network controlled scheme can be accommodated for vertical handover decision in heterogeneous environments.

\section{REFERENCES}

1. Roy, SD \& Reddy, SRV 2014, 'Signal strength ratio based vertical handoff decision algorithms in integrated heterogeneous networks', Wireless personal communications, vol. 77, no. 4, pp. 2565-2585.

2. Lee, DW, Gil, GT \& Kim, DH 2010, 'A cost-based adaptive handover hysteresis scheme to minimize the handover failure rate in 3GPP LTE system', EURASIP Journal on Wireless Communications And Networking, 2010:750173.

3. Peng, Y, Zhang, Y, Kou, G \& Shi, Y 2012, 'A multicriteria decision making approach for estimating the number of clusters in a data set', PLoS One, vol. 7 no. 7, p. 41713.

4. Rathi, R, Khanduja, D \& Sharma 2016, 'Efficiency of fuzzy MADM approach in Six Sigma analysis phase in automotive sector', Journal of Industrial Engineering International, vol. 12, no. 3, pp. 377-387.

5. Miyim, AM, Ismai, LM \& Nordin, R 2014, 'Vertical handover solutions over LTE-advanced wireless networks: An Overview', Wireless Personal Communications, vol. 77, no. 4, pp. 3051-3079.

6. Lee, DW, Gil, GT \& Kim, DH 2010, 'A cost-based adaptive handover hysteresis scheme to minimize the handover failure rate in 3GPP LTE system', EURASIP Journal on Wireless Communications And Networking, 2010:750173.

7. Mehra, L \& Gupta, MK 2013, 'Fuzzy neural networks in vertical handover technology for next generation in wireless networks', International Journal of Advanced Research in Computer Science, vol. 4, no. 3, pp. 145-150.

8. Mohanty, S \& Akyildiz, IF 2006, 'A (Cross-Layer, no. Layer $2+3$ ) handoff management protocol for next-generation wireless systems', IEEE Transactions on Mobile Computing, vol. 5, no. 10, pp. 1347-1360.

9. Jingjing Wang, Deepak Jagtap, Nael Abu-Ghazaleh and Dmitry Ponomarev' Parallel Discrete Event Simulation for Multi-Core Systems: Analysis and Optimization" IEEE Transactions on Parallel and Distributed Systems, Vol. 25, No. 6, June 2014

10. Mahamod. Ismail, Mohamad. Shanudin and Raed. Saqour" Discrete-event Simulation Modeling tool for Routing Process in GPSR Ad Hoc Network Routing Protocol" Proceedings IEEE International Conference on Telecommunications, 2007 